\title{
Recent developments in multi-parametric three-dimensional stress field representation in plates weakened by cracks and notches
}

\author{
P. Lazzarin, M. Zappalorto, F. Berto \\ University of Padua, Department of management and engineering, Stradella San Nicola 3, 36100, Vicenza, Italy
}

\begin{abstract}
The paper deals with the three-dimensional nature and the multi-parametric representation of the stress field ahead of cracks and notches of different shape. Finite thickness plates are considered, under different loading conditions. Under certain hypotheses, the three-dimensional governing equations of elasticity can be reduced to a system where a bi-harmonic equation and a harmonic equation have to be simultaneously satisfied. The former provides the solution of the corresponding plane notch problem, the latter provides the solution of the corresponding out-of-plane shear notch problem. The analytical frame is applied to some notched and cracked geometries and its degree of accuracy is discussed comparing theoretical results and numerical data from 3D FE models.
\end{abstract}

KEYWORDS. Three-dimensional elasticity; Stress fields; Antiplane solution; Plane solution.

\section{INTRODUCTION}

A pioneering analytical framework was proposed by Dougall [1] to evaluate the three-dimensional stress fields in plates; Dougall's method was later applied by Green [2] to the stress analysis of a thick isotropic elastic plate weakened by a cylindrical hole. The same problem was discussed later also by Sternberg and Sadowsky [3] and by Folias and Wang [4]. As common denominator, these works have a sound discussion on the role played by the plate thickness on the hole tip stresses.

As is well known, an approach providing a general solution for three-dimensional problems of elasticity was developed by Papkovich [5] and Neuber [6]. It is based on a general three-dimensional stress function. By using three harmonic functions, the fourth one being equal to zero, and different curvilinear coordinate systems, Neuber was able to provide some solutions for the three-dimensional problem, in particular those related to the axisymmetric hyperboloidal ligament and the axisymmetric ellipsoidal cavity in an infinite elastic body.

Dealing with cracked components, Hartranft and Sih gave the approximate stress fields near the tip of a through crack in a thin elastic plate using a variational principle [7] and a refinement of the Reissner theory [8]; fundamental contributions are also the extension to the three-dimensional crack case [9] of Williams' two dimensional eigenfunction series [10] as well as the application of the Papkovich-Neuber method to the same case [11].

Among the recent papers we remember here the work by Yang and Freund [12] who took advantage of the Kane and Mindlin hypothesis [13] to analyse the state of stress in a tensioned thin elastic plate containing through-cracks. By using the Fourier transform and the Wiener-Hopf technique they demonstrated the existence of a generalised plane strain field at the crack tip, and confirmed Hartranft and Sih's previous findings [7]. The same result was found numerically also by Nakamura and Parks [14]. In parallel, discussing results from 3D finite element analyses on thin cracked elastic plates remotely subjected to mode II anti-symmetrical loading, Nakamura and Parks [15] found that the asymptotic stress fields were characterized by a combination of plane strain mode II and antiplane mode III singular fields. Their analyses put into light, for the first time, the existence of "local induced modes" due to three-dimensional effects. 
In the same period Pook analysed the stress and displacement distributions in three-dimensional plates weakened by cracks and narrow notches [16, 17]. From the results of 3D finite element analyses Pook [17] noted that in a plate under a nominal, far applied, Mode II loading there was a zone, close the notch tip (the notch tip radius being small but different from zero), where Mode III displacements were induced.

The Kane and Mindlin theory was applied by Kotousov and Lew [18] to analyse the stress singularities related to angular corners in plates of arbitrary thickness subjected to in-plane loadings and various boundary conditions. The new singular stress states were referred to as the out-of-plane singularities and the corresponding fracture mode as the out-of-plane singular mode or $\mathrm{K}_{\mathrm{O}}$ - mode.

The intensity of local out-of-plane stress fields at the tip of pointed V-notches in 3D plates under remote mode II loading was widely documented in refs. [19-21] on the basis of detailed three-dimensional FE analyses. Cracks, blunt cracks as well as a variety of notches, sharp and blunt, were considered in these contributions. Also the role played by higher order terms of the stress field was analysed in detail with reference to the crack case. In the presence of a notch tip radius equal to zero, the out-of-plane stress singularity arising at a small distance from the free surfaces, prior to these ones, was found to match that of the sharp V-notch problem under pure antiplane elasticity. When the V-notch opening angle is large enough to make non singular the mode II stress distribution, the induced Mode III stress field remains singular.

The local interaction between loading modes has been recently justified analytically by Lazzarin and Zappalorto [22] for pointed and sharply radiused notches in finite thick plates. On the basis of a generalised plane strain assumption, it was demonstrated that the governing equations of three-dimensional elasticity can be reduced in complexity solving, in combination, a bi-harmonic equation and a harmonic equation. The former provides the solution to the corresponding plane notch problem, the latter that of the antiplane elasticity problem for the same notch geometry. The two equations have to be simultaneously satisfied in a 3D problem, thus justifying theoretically the mutual interaction between mode II and mode III. This result was supported by a number of finite element analyses carried out on mode II loaded thick plates with sharp and blunt notches [22,23]. As a result, some previous closed-form solutions [24,25] obtained for axi-symmetric bodies under torsion can be used to analyse the out-of-plane stress distributions in 3D plates.

Starting from the analytical frame provided in [22], the aim of the present work is to present the 3D stress fields in some cases of practical interest. In the first case the stress fields close to a rectangular hole in a plate of finite thickness under tension is investigated. The second example deals with a finite thickness plate weakened by a crack. Two forces are applied to generate Mode III loading conditions on the plate and the automatically generated coupled Mode II is investigated paying also attention to the scale effect governing the induced mode.

\section{A NEW FRAME FOR THE ANALYSIS OF THE THREE-DIMENSIONAL STRESS FIELD}

new approach to the analysis of the three-dimensional notch problems has been recently proposed in Ref. [22]. According to a generalized plane strain hypothesis, the displacement components are given by:

$$
u_{x}=u(x, y) \quad u_{y}=v(x, y) \quad u_{z}=b z \times w(x, y)
$$

where $\mathrm{z}$ is the through-the-thickness coordinate and $\mathrm{b}$ is a constant term. Doing so, the normal strains $\varepsilon_{\mathrm{xx}}, \varepsilon_{\mathrm{yy}}, \varepsilon_{\mathrm{zz}}$ as well as $\gamma_{\mathrm{xy}}$ are independent of $\mathrm{z}$. Taking advantage of the stress-strain relationships it was demonstrated that also the stress components $\sigma_{\mathrm{xx}}, \sigma_{\mathrm{yy}}, \tau_{\mathrm{xy}}$ and $\sigma_{\mathrm{zz}}$ are independent of $z$, whereas the out-of-plane shear stress components depend on $z$ according to the following expressions:

$$
\tau_{y z}=G \times b z \frac{\partial w}{\partial y} \quad \tau_{x z}=G \times b z \frac{\partial w}{\partial x}
$$

Then the equilibrium equation in the $z$ direction simply gives [22]:

$$
\nabla^{2} w=0
$$

where $\nabla^{2}$ denotes the two-dimensional Laplacian operator. Differently, invoking Eq. (3), the equilibrium equations in the $\mathrm{x}$ and $\mathrm{y}$ directions give:

$$
\frac{\partial^{2} \sigma_{x x}}{\partial x^{2}}+\frac{\partial^{2} \sigma_{y y}}{\partial y^{2}}+2 \frac{\partial^{2} \tau_{x y}}{\partial y \partial x}=0
$$

Eq. (4) is automatically satisfied by the classic Airy stress function $\phi(x, y)$ : 


$$
\sigma_{x x}=\frac{\partial^{2} \varphi}{\partial y^{2}} \quad \sigma_{y y}=\frac{\partial^{2} \varphi}{\partial x^{2}} \quad \tau_{x y}=-\frac{\partial^{2} \varphi}{\partial x \partial y}
$$

At the same time, accounting for the generalised Hooke law for stresses and strains, the in-plane compatibility equation can be written in the form [22]:

$$
\nabla^{4} \varphi=v \nabla^{2} \sigma_{\text {说 }}=0
$$

where the equality to zero is guaranteed by the third of Beltrami-Mitchell's equations.

As a result, the three dimensional notch problem can be converted into a bi-harmonic problem and a harmonic problem being valid the following differential equation system:

$$
\left\{\begin{array}{l}
\nabla^{4} \varphi=0 \\
\nabla^{2} w=0
\end{array}\right.
$$

Here $\mathrm{w}$ and $\phi$ are implicitly defined according to Eqs. (2) and (5), respectively. Note that Eq. (7a) is the common biharmonic equation governing the solution of the plane problem; Eq. (7b) is, instead, the harmonic equation governing the antiplane elasticity problem. It was demonstrated [22, 23] that this new frame is effective not only in the presence of pointed notches (sharp cracks or re-entrant corners) but also in the case of sharply radiused notches. The range of applicability does mainly depend on the notch tip radius.

One should note that, in order to guarantee all the fundamental equations of 3D elasticity, Eq. (7a) and Eq. (7b) must be simultaneously satisfied. This allows to explain an important feature of three-dimensional stress distributions in plates: far applied loads resulting in local skew-symmetric $\phi$ function (mode II stress components) inherently provoke a local skewsymmetric $w$ function (mode III stress components). Viceversa, far applied loads resulting in local skew-symmetric $w$ function (twisting) inherently provoke a local skew-symmetric $\phi$ function (mode II stress components). The same holds valid, obviously, for symmetric loading conditions. Far applied loads resulting in a local symmetric $\phi$ function (mode I stress components) results in a local symmetric $w$ function, which is non-singular also in the presence of a singularity point. This justifies by a theoretical point of view the mutual interaction between loading modes shown numerically in refs. [19-21] and allows to understand why Nisitani and Chen [26] discussed, in principle, about four distinct loading modes. It is also worth mentioning that the proposed solution is not valid on the free surfaces of plates, where some edge effects, like the corner point singularities noted by Benthem [27], might arise. It is valid up to a small distance from the free-surfaces.

In the next sections two examples are provided with the aim to discuss the degree of accuracy of the newly developed three-dimensional theory.

\section{THE SPATIAL RECTANGULAR HOLE PROBLEM}

he plane problem of a plate weakened by a rectangular hole has been analysed by Savin [28]. The mapping function approximating the rectangular hole was constructed on the basis of the Schwarz-Christoffel transformation and the complex functions used to determine the stress state according to the KolosofMuskelisvily method were given explicitely. In this section Savin's two dimensional analysis is extended to the threedimensional case. For more details, see Ref. [23].

In particular, a rectangular hole in a finite thickness plate loaded in tension is considered (figure 1a). The rectangle corner can be regarded as a pointed $\mathrm{V}$-notch of which the bisector is $45^{\circ}$ inclined with respect to the loading direction. As a consequence, the uniaxial remote applied tension induces local in-plane mixed mode stresses (mode I plus mode II), which can be described, according to Eq. (7a), using Williams' plane solution for re-entrant corners.

For a fixed value of the through-the-thickness coordinate, $z$, taking advantage of a polar reference system centred at the notch tip (see figure 1b), the in-plane stresses must vary according to Williams' singularity degrees, $1-\lambda_{1}$ for mode I and 1 $\lambda_{2}$ for mode II; in parallel, the stress field intensities can be quantified by the corresponding Notch Stress Intensity Factors (NSIFs) [29]. However, the three-dimensional nature of the problem induces, besides the in-plane stresses, the out-of-plane shear stress components, $\tau_{\mathrm{zr}}$ and $\tau_{\mathrm{z} \theta}$ which do not belong, by nature, to the Williams solution. These stress components can be obtained by the following w function [26]: 


$$
w=D_{s} r^{\lambda_{3, s}} \cos \left(\lambda_{3, s}\right) \theta+D_{a} r^{\lambda_{3, a}} \sin \left(\lambda_{3, a}\right) \theta
$$

where $\lambda_{3, s}=2 \lambda_{3, a}=\pi / \gamma$, so that only the skew-symmetric part of $\mathrm{w}$ contributes to the singular behaviour of stress fields. Accordingly the antiplane mode III shear stresses close to the tip can be determined as:

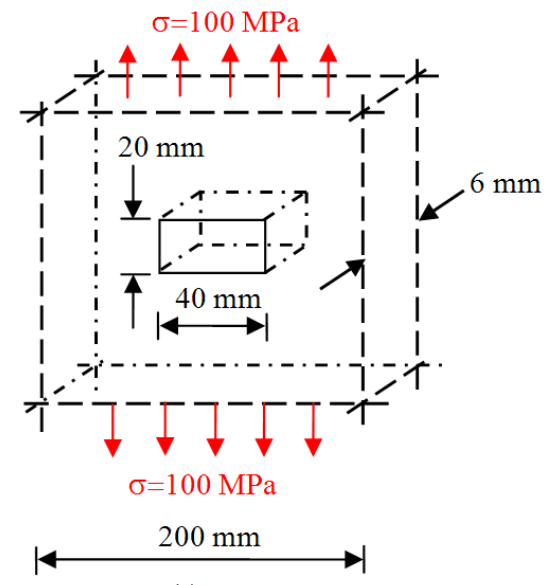

(a)

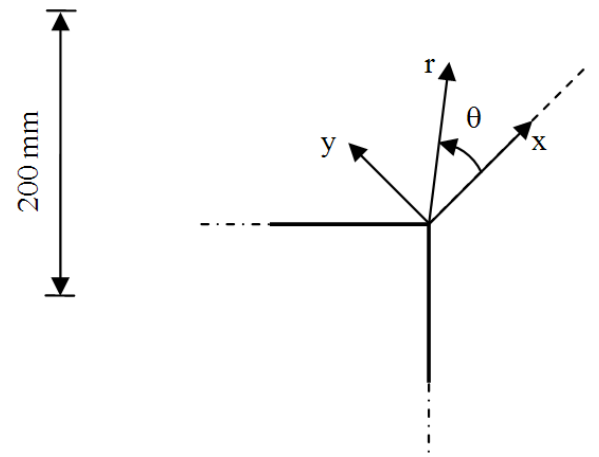

(b)

Figure 1: Thick plate weakened by a rectangular hole under tension (a); coordinate system used for stress components (b).

$$
\tau_{z r}=\frac{K_{3}(\mathrm{z}) r^{\lambda_{3, a}-1}}{\sqrt{2 \pi}} \sin \lambda_{3, a} \theta \quad \tau_{z \theta}=\frac{K_{3}(\mathrm{z}) r^{\lambda_{3, a}-1}}{\sqrt{2 \pi}} \cos \lambda_{3, a} \theta
$$

where:

$$
K_{3}(z)=\lim _{r \rightarrow 0} \sqrt{2 \pi} r^{1-\lambda_{3, a}} \tau_{z \theta}(r, \theta=0)
$$

is the mode III NSIF, to be thought of as the natural extension to the out-of-plane mode of Gross and Mendelson's definitions given for the in-plane modes. In order to validate these theoretical results, a detailed finite element analysis on the geometry shown in figure 1a has been carried out. 20 node brick elements have been used with a very fine mesh pattern, in order to get the desired degree of accuracy. The material has been modelled according to a linear elastic behaviour, with $\mathrm{E}=206000 \mathrm{MPa}$ and $v=0.3$.

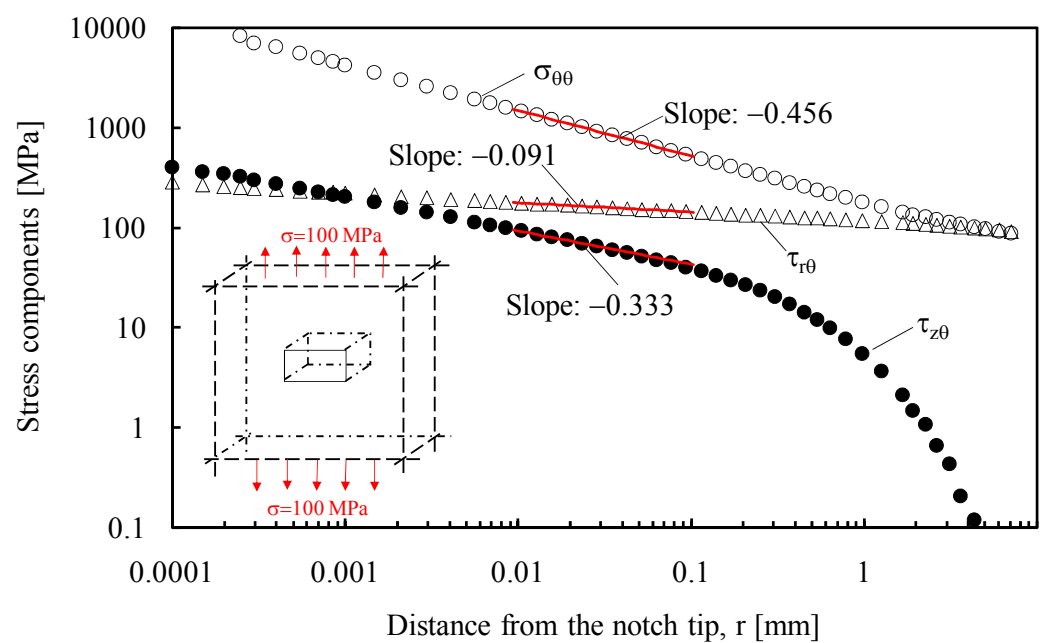

Figure 2: Plots of the stress components $\tau_{\mathrm{z} \theta}, \sigma_{\theta \theta}$ and $\tau_{\mathrm{r} \theta}$ along the notch bisector line of a rectangular hole in a thick plate under tension. Distance from the mid plane $z=2.5 \mathrm{~mm}$. Applied tension $\sigma=100 \mathrm{MPa}$. 


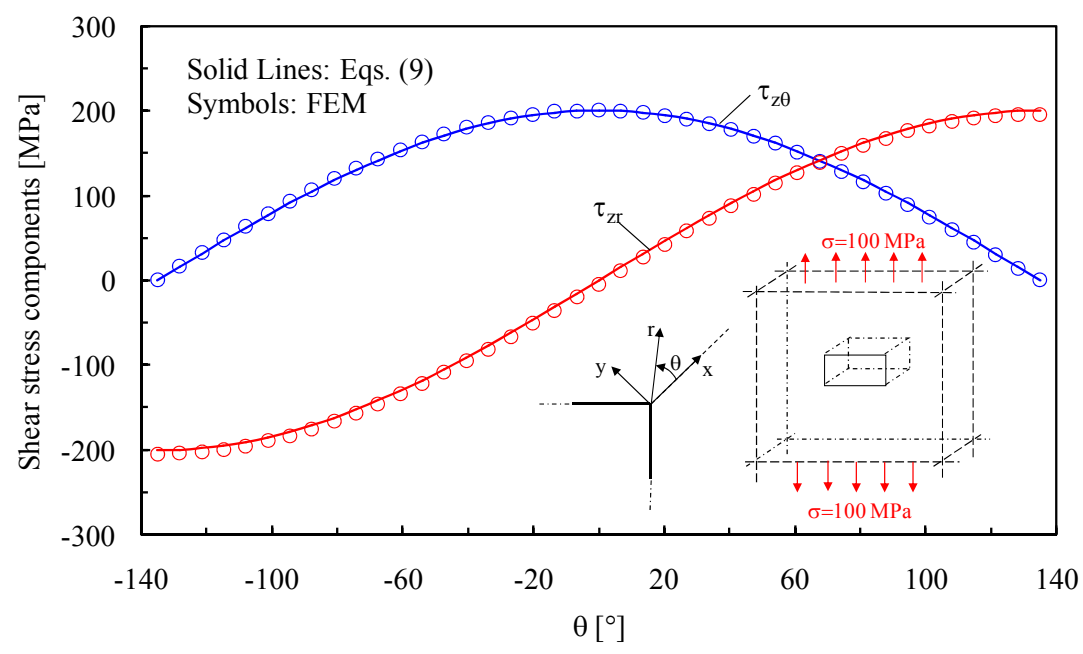

Figure 3: Plots of the stress components $\tau_{z \theta}$ and $\tau_{z \mathrm{r}}$ along a circular path of radius $\mathrm{r}=0.001 \mathrm{~mm}$ centred at the notch tip, $-135^{\circ} \leq \theta \leq 135^{\circ}$, and comparison with Eq. (9). Rectangular hole in a thick plate under tension. Distance from the mid plane $z=2.5$ mm. Nominal stress $\sigma_{\mathrm{n}}=100 \mathrm{MPa}$.

The stresses have been evaluated initially along the notch bisector line, with reference to the distance $z=2.5 \mathrm{~mm}$ from the mid-plane. Results are shown in figure 2 where that the singularity degree of in-plane stresses is shown to be in agreement with the Williams solution. It is worth noting that the stress components $\sigma_{\theta \theta}$, linked to the local mode I, is much higher than the mode III stress component $\tau_{z \theta}$.

Antiplane shear stresses from FEA have been evaluated also on a circular path of radius $\mathrm{r}=0.001 \mathrm{~mm}$ centred at the notch tip and compared to the theoretical prediction based on Eq. (9). The comparison, shown in figure 3, documents a satisfactory agreement.

Figure 4 shows the through the thickness variation of the NSIFs, $\mathrm{K}_{1}, \mathrm{~K}_{2}, \mathrm{~K}_{3}$. It can be seen that the hypothesis postulated in the previous section are verified on the major part of the plate thickness: the in-plane NSIFs are almost constant while $\mathrm{K}_{3}$ varies linearly with $\mathrm{z}$ up to a maximum value which is located in the vicinity of the free surface.

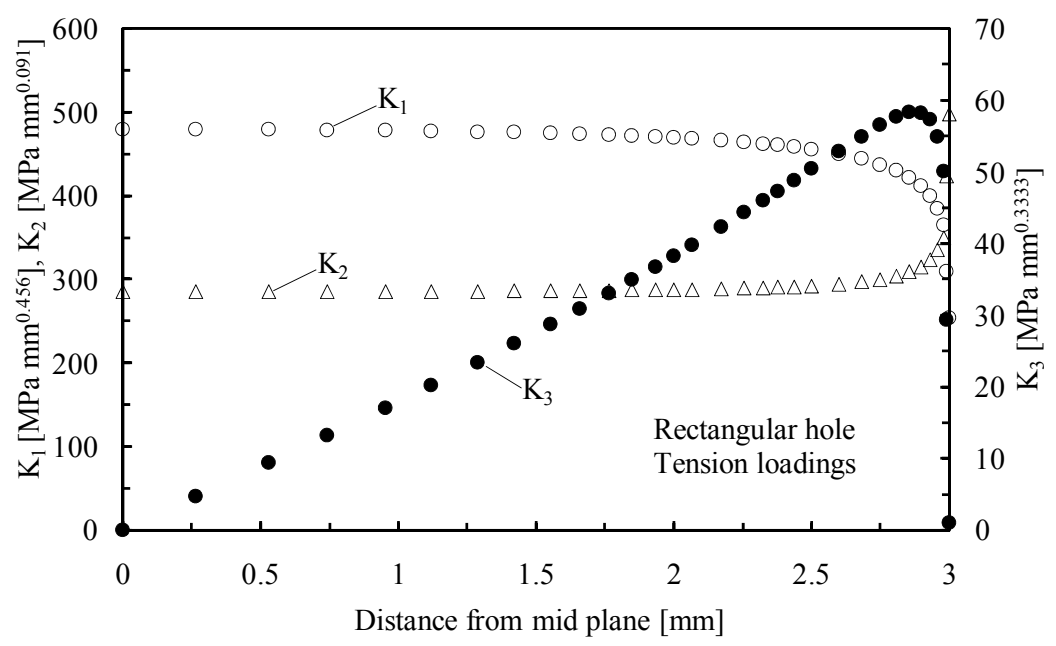

Figure 4. NSIFs plotted along the thickness of the plate. Rectangular hole in a thick plate under tension. Nominal stress $\sigma=100 \mathrm{MPa}$.

\section{D V-NOTCHED PLATE UNDER TORSION LOADING}

I

$\mathrm{n}$ this session another example of coupled mode generation is discussed. A finite thickness plate weakened by a crack has been modeled and two forces have been applied to generate Mode III loading on the plate (see Fig. 5). The values of the stress intensity factors, tied to the generating and the coupled mode and normalized by $\mathrm{K}_{\text {III, appl., defined }}$ 
as the maximum value of $\mathrm{K}_{\mathrm{III}}$ at the mid plane, have been plotted through the plate thickness for different values of the Poisson's ratio (see Fig. 6). As it can be observed from the figure also the limit case $v=0$ has been considered. The figure refers to a distance $\mathrm{x}=0.1 \mathrm{~mm}$ from the crack tip fully included in the zone governed by the leading order terms. The analysis shows that by applying a remote Mode III loading automatically a coupled Mode II is generated in the plate. As well visible from the figure the coupled Mode is only slightly influenced by the Poisson's ratio and that, more important, it arises also when $v=0$.

By considering different plate thicknesses it is possible to investigate the scale effect when an externally applied Mode III induces a coupled Mode II. Figure 7 reports the induced in plane shear stress component $\tau_{\mathrm{xy}}$ for different plate thicknesses $(\mathrm{h}=2.5,10$ and $40 \mathrm{~mm})$. It is evident that decreasing the plate thickness the intensity of the induced stress increases according to the square root of the ratio between the considered plate thicknesses.

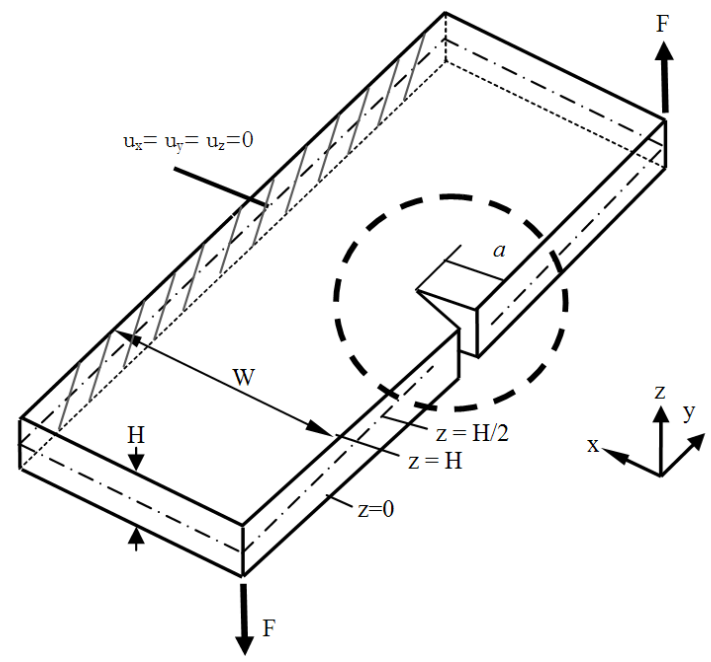

Figure 5: Three-dimensional plate weakened by crack under torsion loading. Two forces have been applied in z-direction while all displacements have been constrained on the dashed surface opposite to the notch.

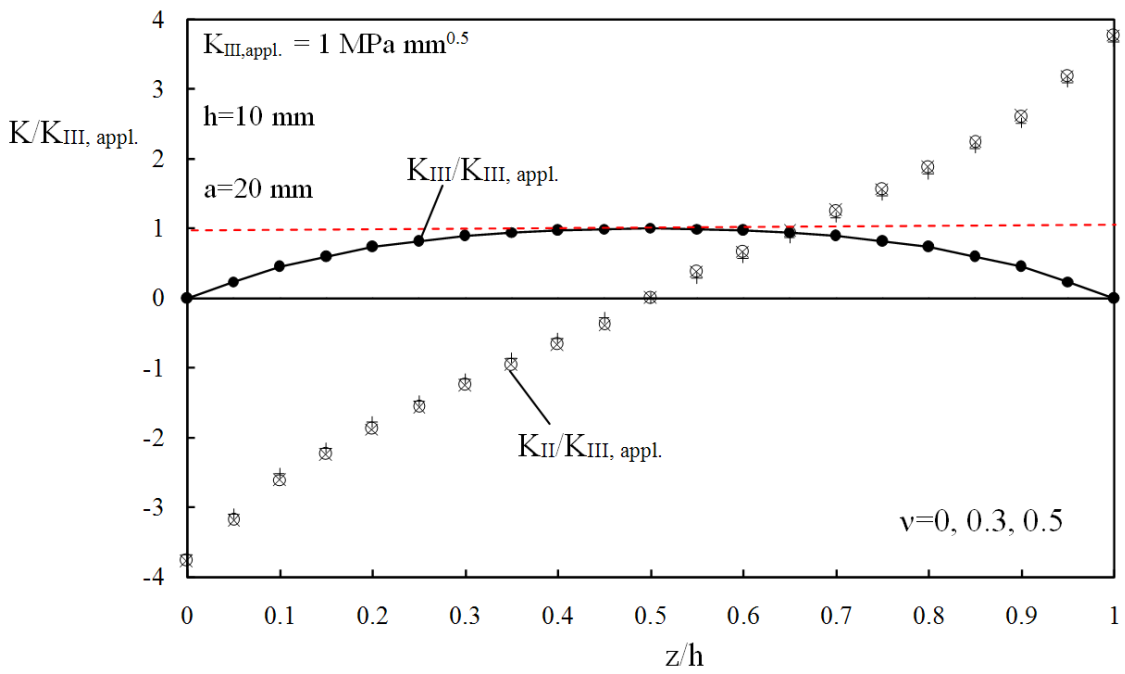

Figure 6: Variations of the stress intensity factors (mode III and coupled mode) along the plate thickness.

\section{CONCLUSIONS}

$\mathrm{I}$ $\mathrm{n}$ this paper a stress field theory for plates of finite thickness is revisited and applied to some cases of practical interest. According to this theory the three-dimensional governing equations of elasticity can be simplified into a biharmonic equation and a harmonic one. The former provides the solution of the corresponding plane notch 
problem, the latter that of the corresponding out-of-plane notch problem. The two mentioned differential equations must be solved simultaneously, thus justifying by a theoretical point of view the mutual interaction between loading modes shown numerically by other authors. The capability of the new frame to describe the local stress fields in plates of arbitrary thickness under various loading conditions has been verified by some practical examples.

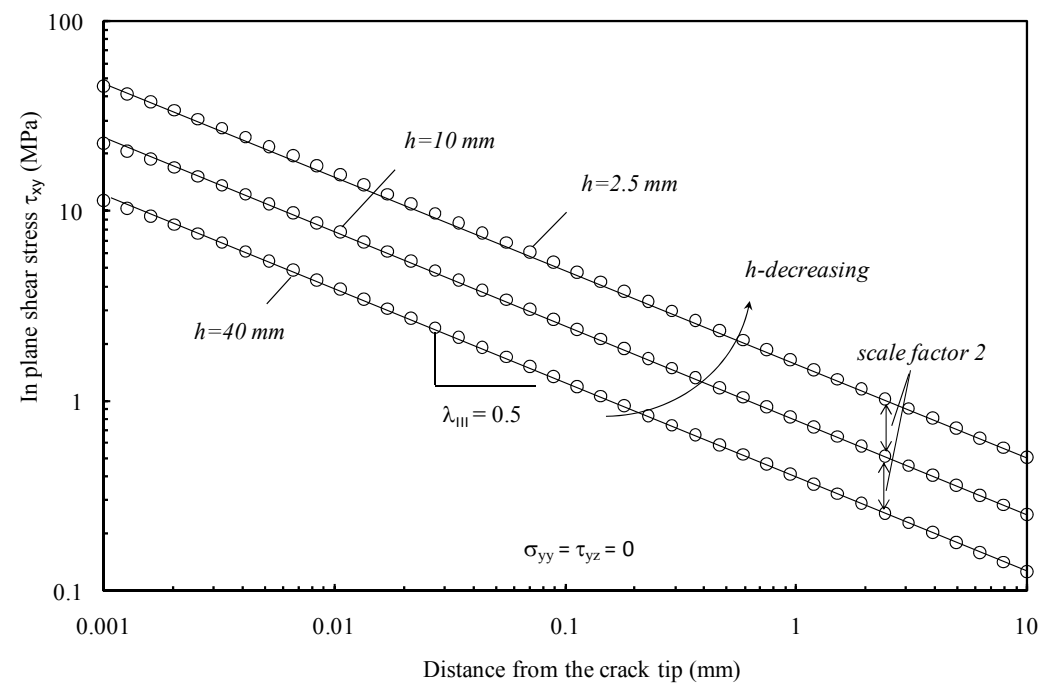

Figure 7: Scale effect on in-plane shear stresses (plate thickness $\mathrm{h}=40,10$ and $2.5 \mathrm{~mm}$ ) induced by Mode III loading with $\mathrm{K}_{\mathrm{III}}=1 \mathrm{MPa}$ $\mathrm{mm}^{0.5}$. $\tau_{\mathrm{xy}}$ was plotted at $\mathrm{z} / \mathrm{h}=0$ (free surface) where the maximum effect due to induced mode II occurs. The plate sizes are scaled by a factor $1 / 4$ while the resulting stresses are scaled by a factor 2 .

\section{REFERENCES}

[1] Dougall, J., An analytical theory of the equilibrium of an isotropic elastic plate, Trans. Roy. Soc., Edinburgh, 41 (1904) 129-228.

[2] Green, A.E., Three-dimensional stress systems in isotropic plates, I. Philos. Trans. R. Soc., London, Ser A 240 (1948) 561-97.

[3] Sternberg, E., Sadowsky, M.D., Three-Dimensional Solution for the Stress Concentration Around a Circular Hole in a Plate of Arbitrary Thickness, J. Appl. Mech., 16 (1949) 27-38.

[4] Folias, E.S., Wang, J-J., On the three-dimensional stress field around a circular hole in a plate of arbitrary thickness. Comput. Mech., 6 (1990) 379-91.

[5] Papkovich, P.F., Solution Générale des équations differentielles fondamentales d'élasticité exprimée par trois fonctions harmoniques, Compt. Rend. Acad. Sci. Paris, 195 (1932) 513-15.

[6] Neuber, H., Theory of notch stresses, Berlin: Springer-Verlag; (1958).

[7] Hartranft, R.J., Sih G.C., An approximate three-dimensional theory of plates with application to crack problems, Int. J. Eng., Science 8 (1970) 711-29.

[8] Hartranft, R.J., Sih G.C., Effect of Plate Thickness on the Bending Stress Distribution Around Through Cracks, J. Math. Phys., 47 (1968) 276-91.

[9] Hartranft, R.J., Sih, G.C., The use of eigenfunction expansions in the general solution of three-dimensional crack problems, J. Math. Mech., 19 (1969) 123-38.

[10] Williams, ML., Stress singularities resulting from various boundary conditions in angular corners of plate in extension, J. Appl. Mech., 19 (1952) 526-534.

[11] Kassir, M.K., Sih G.C., Application of Papkovich-Neuber potentials to a crack problem, Int. J. Solids Struct., 9 (1973) 643-54.

[12] Yang, W., Freund, L.B., Transverse shear effects for through-cracks in an elastic plate, Int. J. Solids Struct., 21 (1985) 977-94.

[13] Kane, T.R., Mindlin, R.D., High frequency extensional vibrations of plates, J. Appl. Mech., 23 (1956) $277-83$.

[14] Nakamura, T, Parks, DM., Three-dimensional stress field near the crack front of a thin elastic plate, J. Appl. Mech., 55 (1988) 805-13. 
[15] Nakamura, T., Parks, D.M., Antisymmetrical 3-D stress field near the crack front of a thin elastic plate, Int. J. Solids Struct, 25 (1989)1411-26.

[16] Pook, L.P., Some implications of corner point singularities, Eng. Fract. Mech., 48 (1994) 367-78.

[17] Pook, L.P., Finite element analysis of corner point displacements and stress intensity factors for narrow notches in square sheets and plates, Fatigue Fract. Engng. Mater. Struct., 23 (2000) 979-92.

[18] Kotousov, A., Lew, Y.T., Stress singularities resulting from various boundary conditions in angular corners of plates of arbitrary thickness in extension. Int. J. Solids. Struct., 43 (2006) 5100-09.

[19] Berto, F., Lazzarin, P., Harding, S., Kotousov, A., Out-of-plane singular stress fields in V-notched plates and welded lap joints induced by in-plane shear load conditions, Fatigue Fract. Engng. Mater. Struct., 34 (2011) 291-304.

[20] Berto, F, Lazzarin, P, Kotousov, A, Pook, L., On mode O existence at the tip of blunt cracks and U-notches under inplane shear loading, Fatigue Fract. Engng. Mater. Struct., 35 (2012) 538-555.

[21] Berto, F., Lazzarin, P., Kotousov, A., On higher order terms and out-of-plane singular mode, Mech. Mater., 43 (2011) $332-342$

[22] Lazzarin, P., Zappalorto, M., A three-dimensional stress field solution for pointed and sharply radiused V-notches in plates of finite thickness, Fatigue Fract. Engng Mater. Struct. 35 (2012) 1105-19.

[23] Zappalorto, M., Lazzarin, P., Three-dimensional elastic stress fields ahead of notches in thick plates under various loading conditions, Eng. Fract. Mech., accepted for publication.

[24] Zappalorto, M., Lazzarin, P., Filippi, S., Stress field equations for U and blunt V-shaped notches in axisymmetric shafts under torsion, Int. J. Fract. 164 (2010) 253-69.

[25] Zappalorto, M., Lazzarin, P., Stress fields due to inclined notches and shoulder fillets in shafts under torsion, J. Strain. Anal. Eng. Des., 46 (2011) 187-99.

[26] Chen, D., Nisitani, H., Singular stress field near a corner of jointed dissimilar materials under antiplane loads. Bull Japan Soc. Mech. Eng., 35 (1992) 399-403.

[27] Benthem, J.P., State of stress at the vertex of a quarter-infinite crack in half space. Int. J. Solids Struct. 13 (1977) 479_ 92.

[28] Savin, G.N., Stress concentration around holes. New York; Pergamon Press, (1961).

[29] Gross, R., Mendelson A., Plane Elastostatic Analysis of V-Notched Plates. Int. J. Fract. Mech., 8 (1972) 267-276. 